\title{
Dispensações de psicotrópicos anorexígenos no município de Juiz de Fora, Minas Gerais, Brasil
}

\author{
Prescriptions for anorectic psychotropic drugs \\ in the municipality of Juiz de Fora, Minas Gerais, Brazil
}

Eduardo Luiz Mendonça Martins ${ }^{1}$

Maria da Penha Henriques do Amaral ${ }^{1}$

Maria Beatriz Cardoso Ferreira ${ }^{2}$

Alessandra Ésther de Mendonça ${ }^{1}$

Mônica Cecília Santana Pereira ${ }^{3}$

Denise Campos Pereira ${ }^{3}$

Paula Maria Ribeiro

Larissa Pereira Brumano ${ }^{1}$

${ }^{1}$ Universidade Federal de Juiz de Fora (UFJF). Caixa Postal 138. 36307-970 São João del-Rei MG. emartins2br@yahoo.com.br ${ }^{2}$ Departamento de

Farmacologia, Instituto de Ciências Básicas da Saúde, Universidade Federal do Rio Grande do Sul (UFRGS).

${ }^{3}$ Vigilância Sanitária, Prefeitura de Juiz de Fora.

\begin{abstract}
Obesity is a serious public health issue of epidemic proportions. Although drug therapy is one of the therapeutic approaches, it should be preceded by a change in eating habits along with regular exercise. This study assessed prescription of such drugs in 2009, in Juiz de Fora, Minas Gerais, Brazil. Data were collected from consumption bulletins sent monthly to the municipal sanitary surveillance agency (VISA), and from the Brazilian National Management System of Controlled Products. Of the 7,759 notifications assessed, 93.3\% were dispensed by prescription pharmacies and $6.7 \%$ by drugstores. Of that total, $55.4 \%$ were for amphepramone, $33.1 \%$ for femproporex, and $11.5 \%$ for mazindol. The three drugs had a larger consumption rate in the municipality (daily defined dose/1,000 inhabitants/day) than in the country as a whole, during the period under study. The professional with the highest prescription rate was responsible for 3,535 prescriptions. All of these were dispensed by prescription pharmacies, a single outlet being responsible for $99.5 \%$ of these prescriptions. This scenario shows that anorectic psychotropic drug prescription is a relevant public health issue and the criteria for monitoring the prescription and consumption of these drugs should be re-evaluated.
\end{abstract}

Key words Prescriptions, Anorectic drugs, Obesity, Rational drug use
Resumo A obesidade é um grave problema de saúde pública, com prevalência crescente em níveis epidêmicos. Tratamento farmacológico é uma das abordagens terapêuticas, mas deve ser precedido de mudança de hábitos alimentares e prática regular de atividades físicas. Há preocupação com o uso não racional de medicamentos anorexígenos, independente da condição de doença. O estudo avaliou as dispensações destes psicotrópicos realizadas em 2009, Juiz de Fora, MG. Realizou-se a pesquisa em boletins de consumo, enviados mensalmente à VISA municipal, e em registros obtidos no SNGPC. De 7.759 notificações pesquisadas, 93,3\% foram dispensadas por farmácias magistrais e $6,7 \%$ por drogarias. Daquele total, 55,4\% foram de anfepramona, 33,1\% de femproporex e $11,5 \%$ de mazindol. Com base em relatório da Anvisa, as três substâncias apresentaram consumo maior no município (DDD/mil hab/ano) do que no país como um todo, para o mesmo período. O profissional com maior número de receitas aviadas teve 3.535 dispensações. Todas foram dispensadas em farmácias, sendo 99,5\% em um único estabelecimento. O cenário mostra a dispensação de anorexígenos como problema relevante de saúde coletiva e indica a necessidade de reavaliação dos critérios de monitoramento de sua prescrição, dispensação e consumo.

Palavras-chave Dispensação, Anorexígenos, Obesidade, Uso racional de medicamentos 


\section{Introdução}

Associada com alto risco de morbidade e letalidade, a obesidade é grave problema de saúde pública em nosso país, com sua prevalência crescendo em níveis epidêmicos ${ }^{1,2}$. Sendo assim, emerge a necessidade de melhorar a qualidade e a eficácia dos tratamentos disponíveis ${ }^{3,4}$.

O cerne do tratamento atual da obesidade baseia-se em terapias comportamentais, dirigidas para a modificação de atividades e hábitos relacionados à alimentação, exercício, para aumentar o gasto calórico, e orientações nutricionais, para diminuir o consumo de calorias e, particularmente, de gordura. $\mathrm{O}$ uso de fármacos é considerado terapia adjuvante no tratamento da obesidade ${ }^{5}$.

Só há indicação de tratamento medicamentoso se houver falha terapêutica associada às seguintes situações ${ }^{5}$ : (a) pacientes com IMC igual ou superior a $30 \mathrm{~kg} / \mathrm{m}^{2}$; (b) pacientes com IMC igual ou superior a $25 \mathrm{~kg} / \mathrm{m}^{2}$, associado a outros fatores de risco, como hipertensão arterial, Diabetes Mellitus (DM) tipo 2, hiperlipidemia, apneia do sono, osteoartrose, gota, entre outras; (c) pacientes com circunferência abdominal maior ou igual a $102 \mathrm{~cm}$ (homens) e $88 \mathrm{~cm}$ (mulheres).

Existem atualmente, no Brasil, cinco substâncias registradas com indicação para tratamento da obesidade: Anfepramona (Dietilpropiona), Femproporex, Mazindol, Sibutramina e Orlistate. As quatro primeiras são classificadas como substâncias psicotrópicas anorexígenas, fazendo parte da "Lista B2" da Portaria no. 344/1998 (atualizada pela RDC no. 7 de 26 de fevereiro de 2009) da Agência Nacional de Vigilância Sanitária Anvisa ${ }^{6}$. Já o Orlistat age inibindo lípases pancreáticas, reduzindo em 30\% a absorção das gorduras ingeridas ${ }^{7}$. Seu uso deve ser cuidadosamente monitorado, e, segundo a OMS, o prazo máximo recomendado para emprego de derivados anfetamínicos é de quatro meses ${ }^{8}$.

Em 24 de fevereiro de 2011 a Anvisa abriu consulta pública para proibir a comercialização dos anorexígenos psicotrópicos Femproporex, Anfepramona, Mazindol e Sibutramina. Como não houve consenso, ainda aguarda-se definição do órgão regulador com relação à permissão de venda desses produtos.

Previamente, em função do uso abusivo de determinados medicamentos no Brasil, foi editada e publicada a RDC no 27 de 30 de março de $2007^{9}$, que instituiu o Sistema Nacional para Gerenciamento de Produtos Controlados (SNGPC). O SNGPC consiste de sistema informati- zado, com transmissão dos dados por meio de pacotes em formato XML (Extensible Markup Language) - linguagem com recomendação internacional, para gerar padronização em informações. A partir de 2008, a implantação foi gradual, obedecendo a cronograma previamente estipulado. O SNGPC para farmácias e drogarias é a primeira etapa de um projeto maior, integrante da Política da Agência Nacional de Vigilância Sanitária, que deverá englobar toda a cadeia de produção (indústrias e distribuidoras), bem como promover o uso racional dos medicamentos que podem causar dependência física e/ou psíquica. O SNGPC tem como principais objetivos: monitorar a dispensação de medicamentos e substâncias entorpecentes e psicotrópicas e seus precursores; otimizar o processo de escrituração; permitir o monitoramento de hábitos de prescrição e consumo de substâncias controladas em determinada região, para propor políticas de controle; captar dados que permitam a geração de informação atualizada e fidedigna para o Serviço Nacional de Vigilância Sanitária (SNVS), para a tomada de decisão; dinamizar as ações da vigilância sanitária ${ }^{10}$

Nesse sentido, o presente estudo teve como finalidade verificar o consumo de medicamentos psicotrópicos anorexígenos na população de Juiz de Fora, Minas Gerais, durante o ano de 2009, por meio da análise de frequências de suas dispensações. Avaliou-se, ainda, a funcionalidade do SNGPC na monitoração da dispensação desses fármacos.

\section{Métodos}

Foi realizado estudo observacional descritivo, de corte transversal retrospectivo, abrangendo o município de Juiz de Fora. Este se situa na mesorregião do sudeste do estado de Minas Gerais, denominada Zona da Mata Mineira. Tem população de 517.872 habitantes ${ }^{11}$. Possui alto grau de urbanização, com $98,8 \%$ da sua população residindo na área urbana. É o quarto município mais populoso de Minas Gerais e o $36^{\circ}$ do Brasil. As mulheres representam $52,4 \%$ da população. Seu Índice de Desenvolvimento Humano (IDH), em 2000 , era de 0,828 , o nono melhor do estado Possui grande área de influência, por ser a cidade mais populosa da região, além de concentrar diversas instituições de ensino superior, muitas indústrias e diversificado setor terciário. Sua abrangência se estende desde cidades do sul de Minas até do centro-oeste fluminense, além de diversos 
Foram utilizados dados provenientes do Sistema Nacional para Gerenciamento de Produtos Controlados (SNGPC), mediante prévia solicitação ao setor de Gerência Geral de Medicamentos (GGMED) / Coordenação do Sistema Nacional de Gerenciamento de Produtos Controlados (CSGPC) da Anvisa. Foram analisados, ainda, dados enviados à VISA Municipal, por meio da Relação Mensal de Notificações de Receita "B2" (RMNRB2), constituída por informações fornecidas mensalmente à Vigilância Sanitária Municipal pelos estabelecimentos.

Com base em SNGPC e RMNRB2, foram coletadas as seguintes informações para análise: estabelecimento farmacêutico (identificação e tipo), medicamento anorexígeno dispensado, sua quantidade e concentração, mês da dispensação e CRM do profissional prescritor.

\section{Amostra}

Dos 171 estabelecimentos farmacêuticos (drogarias e farmácias) cadastrados em julho de 2009, na Vigilância Sanitária da Secretaria Municipal de Saúde da Prefeitura de Juiz de Fora, 17 não comercializavam psicotrópicos e 37 estavam com pendências para habilitação junto ao SNGPC (processos em andamento ou ainda não iniciados). Portanto, 117 estabelecimentos já trabalhavam com o SNGPC, constituindo o universo de pesquisa.

Dos 117 estabelecimentos, 83 eram drogarias e 34 farmácias. Desse universo, foram selecionados, segundo informações da VISA municipal, com base nos históricos do SNGPC e nas Relações Mensais de Notificações de Receita “B2” (RMNRB2), enviadas mensalmente durante todo o ano de 2009, os estabelecimentos que realizavam maior quantidade de dispensações de psicotrópicos. A amostra ficou, então, constituída por 16 estabelecimentos (8 farmácias e 8 drogarias), representando, aproximadamente, $60 \%$ do movimento de dispensação de anorexígenos psicotrópicos no período estudado. Utilizou-se o mesmo número de drogarias e farmácias, para avaliar se havia diferença entre os dois tipos de estabelecimento.

Foram incluídas na análise as dispensações de todas as prescrições dos psicotrópicos anore-

xígenos (Lista B2 da Portaria 344/1998) ${ }^{6}$ anfepramona, femproporex e mazindol efetuadas pelos estabelecimentos selecionados, no período de janeiro a dezembro de 2009. No presente estudo, não foram incluídos os psicotrópicos Sibutramina e Fentermina. A primeira ainda não pertencia à lista B2 no período estudado. Foi posteriormente reclassificada, passando da Lista C1 para a Lista B2, em março de $2010^{12}$. A fentermina, por sua vez, apesar de citada na RDC n ${ }^{\circ} .58$ de $2007^{13}$, não aparece nos relatórios de consumo de anorexígenos enviados pelos estabelecimentos, no período estudado.

\section{Aspectos éticos}

O projeto foi aprovado pelo Comitê de Ética em Pesquisa (CEP) da Universidade Federal de Juiz de Fora (UFJF). Foi obtido parecer favorável para acesso aos dados do município, nas duas instâncias da Vigilância Sanitária (municipal e federal).

\section{Análise dos dados}

Os estabelecimentos foram divididos em farmácias e drogarias, conforme descrito anteriormente: 8 farmácias (codificadas por números: 1 a 8 ) e 8 drogarias (codificadas também por números: 9 a 16).

Os dados dos prescritores (número de registro no Conselho Profissional, codificado posteriormente para manutenção do sigilo) foram comparados com os do cadastro do Conselho Regional de Medicina de Minas Gerais, para avaliar se esses profissionais estavam devidamente registrados e em qual a especialidade. Os dados foram obtidos por meio de endereço eletrônico disponível para pesquisa pública ${ }^{14}$.

Os medicamentos foram avaliados, registrando a substância prescrita (por meio da Denominação Comum Brasileira - DCB $)^{15}$, a quantidade dispensada (fornecida) e a concentração (em miligramas).

Para quantificar o uso de medicamentos, este estudo adotou como unidade de medida a DDD do sistema Anatomical Therapeutical Chemical / Defined Daily Doses (ATC/DDD). Para os cálculos de DDD/mil habitantes/dia, os resultados foram obtidos utilizando a seguinte fórmula:

\begin{tabular}{|c|c|}
\hline \multirow{2}{*}{$\begin{array}{c}\mathrm{DDD} / \mathrm{mil} \\
\text { habitantes/dia }=\end{array}$} & $\begin{array}{l}\text { Consumo anual da } \\
\text { substância x } 1.000\end{array}$ \\
\hline & $\begin{array}{l}\text { DDD da substância } \\
\text { x população x } 365 \text { dias }\end{array}$ \\
\hline
\end{tabular}


Os dados coletados foram registrados em planilha eletrônica do Microsoft Office Excel 2007 e analisados no pacote estatístico Statistical $\mathrm{Pa}$ ckage for the Social Sciences (SPSS) versão 14.0. Foi realizada estatística descrita, por meio do cálculo de frequências absolutas e relativas das variáveis em estudo.

\section{Resultados}

O acesso ao SNGPC mostrou-se bastante lento, principalmente em horários de pico comercial (de 10 às 12 horas e de 14 às 17 horas). Em diversos momentos esteve inacessível, para manutenção do banco de dados ou ajustes do sistema. Durante a pesquisa, verificaram-se alguns registros sem consistência, com finalização e abertura de inventário sem justificativas, estoques negativos e incompatíveis com as saídas. Alguns relatórios gerados pelo SNGPC se mostraram incompletos e inconsistentes. Estabelecimentos que tinham dispensado anorexígenos no período do estudo, conforme RMNRB2, apresentavam histórico sem movimentação no sistema. Nesses casos, foi necessário recorrer aos registros da RMNRB2, enviada periodicamente à VISA municipal pelas farmácias e drogarias.

Dos dezesseis estabelecimentos analisados, catorze se localizavam no centro da cidade. Apenas uma farmácia e uma drogaria se localizavam em um bairro da zona sul da cidade. Das oito drogarias, seis faziam parte de uma mesma rede comercial, com vários estabelecimentos na cidade e região.

Durante a pesquisa, obtiveram-se os registros de 7.832 prescrições. Destas, 73 (1,1\%) apresentaram erros em pelo menos um dos campos, sendo excluídas da análise. Foram validadas, assim, as informações de 7.759 prescrições no período estudado.

Quanto aos erros encontrados, em 11 prescrições constavam CRM de médicos transferidos de Minas Gerais para outros estados, entre 1959 e 2007, todos com números distintos. Em 16, constavam CRM de médicos falecidos antes de 2008, sendo 13 com um mesmo CRM, todas emitidas no mesmo estabelecimento. Em 10, constavam CRM com valores acima de 52.600, sendo que, somente em janeiro de 2010, o CRM emitiu o cadastro número 51.000. Destas, nove foram emitidas pelo mesmo estabelecimento. Em 7, constavam números de CRM cancelados em Minas Gerais, entre 1982 e 2006, todos com números distintos. Em 21, não havia informação do CRM do prescritor. Em 8, as prescrições não continham informação da quantidade dispensada.

Na Tabela 1, registrou-se a quantidade de prescrições aviadas por estabelecimento, divididas por número de prescrições e quantidade de cada fármaco (em mg). Do total de prescrições, 4.299 $(55,4 \%)$ eram de anfepramona, $2.567(33,1 \%)$ de femproporex e $893(11,5 \%)$ de mazindol. Existiu um predomínio dos estabelecimentos 1 e 2 , sendo juntos responsáveis por $72,3 \%(48,1 \%$ da farmácia 1 e $24,2 \%$ da farmácia 2) de todas as dispensações de anorexígenos pesquisadas nesse período. Observa-se, ainda, que a farmácia 1 aviou 81,9\% da quantidade de mazindol dispensada no período e mais da metade $(52,8 \%)$ da quantidade de femproporex. Todos os estabelecimentos dispensaram femproporex e anfepramona, em maior ou menor quantidade. Apenas mazindol não foi dispensado em alguns estabelecimentos.

Comparando-se os dados entre farmácias e drogarias, nota-se que, de todas as prescrições avaliadas, 93,3\% foram dispensadas por farmácias de manipulação e somente $6,7 \%$ por drogarias (Tabela 2). Essa diferença chega a $98,6 \%$ de produtos manipulados versus $1,7 \%$ de industrializados, quando se analisou a quantidade em miligramas de mazindol vendido pelos estabelecimentos estudados.

Cento e oitenta e nove profissionais prescreveram as 7.759 dispensações analisadas. Houve registro de apenas uma prescrição para 93 profissionais $(49,2 \%)$ e de 2 a 10 prescrições para 65 médicos $(34,4 \%)$ (Gráfico 1). Apenas sete médicos emitiram mais de 100 prescrições (cada um), em 2009. Pode-se observar a existência de dois grupos de profissionais - 182 médicos (96,3\%) emitiram até 100 prescrições, em 2009, e 7 profissionais $(3,6 \%)$ prescreveram 101 ou mais vezes, nesse mesmo período.

Para cada registro de CRM obtido, foi definida uma especialidade médica, segundo informação pública, acessada no site do CRM-MG. Apenas seis profissionais possuíam mais de uma especialidade, sendo que, destes, apenas um prescreveu seis vezes no decorrer de 2009; os outros cinco o fizeram apenas uma vez. Entre as prescrições avaliadas, a grande maioria foi emitida por médicos que não tinham especialidade cadastrada no CRM (57\%). Em seguida, apareceram os endocrinologistas, com 35\% das prescrições, e os cardiologistas, com 3,2\%. Apareceram especialidades pouco habituais no tratamento da obesidade, como Ginecologia $(2,7 \%)$, Cirurgia Geral $(0,5 \%)$, Medicina do Trabalho $(0,3 \%)$, Otorrinolaringologia $(0,1 \%)$, Anestesiologia $(0,1 \%)$, Pediatria $(0,1 \%)$ e Derma- 
Tabela 1. Número de prescrições e quantidade de cada fármaco (em mg), por estabelecimento farmacêutico.

\begin{tabular}{|c|c|c|c|c|c|c|c|c|}
\hline \multirow{2}{*}{$\begin{array}{l}\text { Estab } \\
\text { (Tipo) }\end{array}$} & \multicolumn{4}{|c|}{ Femproporex } & \multicolumn{4}{|c|}{ Anfepramona } \\
\hline & $\mathrm{N}$ & $(\%)$ & $\mathrm{mg}$ & $(\%)$ & $\mathbf{N}$ & $(\%)$ & $\mathrm{mg}$ & $(\%)$ \\
\hline 1 - Farm & 1.243 & $(48,4)$ & 1.697 .900 & $(52,8)$ & 1.243 & $(48,4)$ & 1.697 .900 & $(52,8)$ \\
\hline 2 - Farm & 534 & $(20,8)$ & 695.805 & $(21,6)$ & 534 & $(20,8)$ & 695.805 & $(21,6)$ \\
\hline $3-$ Farm & 101 & $(3,9)$ & 126.200 & $(3,9)$ & 101 & $(3,9)$ & 126.200 & $(3,9)$ \\
\hline 4 - Farm & 179 & $(7,0)$ & 227.440 & $(7,1)$ & 179 & $(7,0)$ & 227.440 & $(7,1)$ \\
\hline 5 - Farm & 132 & $(5,1)$ & 170.400 & $(5,3)$ & 132 & $(5,1)$ & 170.400 & $(5,3)$ \\
\hline 6 - Farm & 33 & $(1,3)$ & 39.900 & $(1,2)$ & 33 & $(1,3)$ & 39.900 & $(1,2)$ \\
\hline 7 - Farm & 3 & $(0,1)$ & 4.500 & $(0,1)$ & 3 & $(0,1)$ & 4.500 & $(0,1)$ \\
\hline 9 - Drog & 41 & $(1,6)$ & 30.750 & $(1,0)$ & 41 & $(1,6)$ & 30.750 & $(1,0)$ \\
\hline $10-$ Drog & 53 & $(2,1)$ & 39.750 & $(1,2)$ & 53 & $(2,1)$ & 39.750 & $(1,2)$ \\
\hline $11-$ Drog & 47 & $(1,8)$ & 35.250 & $(1,1)$ & 47 & $(1,8)$ & 35.250 & $(1,1)$ \\
\hline $12-$ Drog & 24 & $(0,9)$ & 18.000 & $(0,6)$ & 24 & $(0,9)$ & 18.000 & $(0,6)$ \\
\hline 8 - Farm & 38 & $(1,5)$ & 28.500 & $(0,9)$ & 38 & $(1,5)$ & 28.500 & $(0,9)$ \\
\hline 13 - Drog & 30 & $(1,2)$ & 22.500 & $(0,7)$ & 30 & $(1,2)$ & 22.500 & $(0,7)$ \\
\hline $14-$ Drog & 39 & $(1,5)$ & 29.250 & $(0,9)$ & 39 & $(1,5)$ & 29.250 & $(0,9)$ \\
\hline 15 - Drog & 37 & $(1,4)$ & 27.750 & $(0,9)$ & 37 & $(1,4)$ & 27.750 & $(0,9)$ \\
\hline 16 - Drog & 33 & $(1,3)$ & 24.750 & $(0,8)$ & 33 & $(1,3)$ & 24.750 & $(0,8)$ \\
\hline Total & 2.567 & - & 3.218 .645 & - & 2.567 & - & 3.218 .645 & - \\
\hline \multirow{2}{*}{$\begin{array}{l}\text { Estab } \\
\text { (Tipo) }\end{array}$} & \multicolumn{4}{|c|}{ Mazindol } & \multicolumn{4}{|c|}{ Total de prescrições } \\
\hline & $\mathrm{N}$ & $(\%)$ & $\mathrm{mg}$ & $(\%)$ & & $\mathrm{N}$ & (\%) & \\
\hline $1-$ Farm & 709 & $(79,4)$ & 55.196 & $(81,9)$ & & 3.731 & $(48,1)$ & \\
\hline 2 - Farm & 65 & $(7,3)$ & 4.400 & $(6,5)$ & & 1.875 & $(24,2)$ & \\
\hline 3 - Farm & 53 & $(5,9)$ & 4.210 & $(6,2)$ & & 533 & $(6,9)$ & \\
\hline 4 - Farm & 7 & $(0,8)$ & 450 & $(0,7)$ & & 437 & $(5,6)$ & \\
\hline 5 - Farm & 26 & $(2,9)$ & 1.725 & $(2,6)$ & & 326 & $(4,2)$ & \\
\hline $6-$ Farm & 2 & $(0,2)$ & 180 & $(0,3)$ & & 186 & $(2,4)$ & \\
\hline 7 - Farm & 0 & $(0,0)$ & 0 & $(0,0)$ & & 92 & $(1,2)$ & \\
\hline $9-$ Drog & 7 & $(0,8)$ & 280 & $(0,4)$ & & 90 & $(1,2)$ & \\
\hline $10-$ Drog & 3 & $(0,3)$ & 120 & $(0,2)$ & & 82 & $(1,1)$ & \\
\hline $11-$ Drog & 4 & $(0,4)$ & 160 & $(0,2)$ & & 70 & $(0,9)$ & \\
\hline 12 - Drog & 8 & $(0,9)$ & 280 & $(0,4)$ & & 69 & $(0,9)$ & \\
\hline 8 - Farm & 3 & $(0,3)$ & 120 & $(0,2)$ & & 65 & $(0,8)$ & \\
\hline 13 - Drog & 6 & $(0,7)$ & 240 & $(0,4)$ & & 57 & $(0,7)$ & \\
\hline 14 - Drog & 0 & $(0,0)$ & 0 & $(0,0)$ & & 53 & $(0,7)$ & \\
\hline 15 - Drog & 0 & $(0,0)$ & 0 & $(0,0)$ & & 50 & $(0,6)$ & \\
\hline 16 - Drog & 0 & $(0,0)$ & 0 & $(0,0)$ & & 43 & $(0,6)$ & \\
\hline Total & 893 & - & 67.361 & - & & 7.759 & - & \\
\hline
\end{tabular}

Estab $=$ Estabelecimento $;$ Farm $=$ Farmácia Drog $=$ Drogaria

tologia $(0,1 \%)$. Ressalta-se, ainda, a existência de prescrição de agentes anorexígenos por endoscopista, radiologista, urologista e oftalmologista.

Na Tabela 3, analisou-se a associação entre os 20 profissionais com maior número de prescrições e as farmácias em que as mesmas foram aviadas. Das 3.535 prescrições de psicotrópicos anorexígenos emitidas pelo profissional "A", 99,5\% (3.518) foram dispensadas pelo estabelecimento 1. As 17 restantes foram dispensadas pelas farmá- 
Tabela 2. Número de prescrições e quantidade de cada fármaco (em mg), por tipo de estabelecimento farmacêutico.

\begin{tabular}{|c|c|c|c|c|c|c|c|c|c|}
\hline \multirow[b]{2}{*}{ Estab. } & \multicolumn{4}{|c|}{ Femproporex } & \multicolumn{5}{|c|}{ Anfepramona } \\
\hline & $\mathrm{N}$ & $(\%)$ & mg & $(\%)$ & $\mathbf{N}$ & $(\%)$ & & mg & $(\%)$ \\
\hline Farmácias & 2.266 & $(88,3)$ & 2.992 .895 & $(93,0)$ & 4.110 & $(95,6)$ & 13.4 & 426.775 & $(97,7)$ \\
\hline Drogarias & 301 & $(11,7)$ & 225.750 & $(7,0)$ & 189 & $(4,3)$ & & 319.250 & $(2,3)$ \\
\hline \multirow[t]{2}{*}{ Total } & 2.567 & - & 3.218 .645 & - & 4.299 & - & 13.7 & 746.025 & - \\
\hline & \multicolumn{4}{|c|}{ Mazindol } & \multicolumn{5}{|c|}{ Total de prescrições } \\
\hline Estab. & $\mathbf{N}$ & $(\%)$ & $\mathrm{mg}$ & $(\%)$ & & & $\mathbf{N}$ & $(\%)$ & \\
\hline Farmácias & 869 & $(97,3)$ & 66.441 & $(98,6)$ & & & 245 & $(93,3)$ & \\
\hline Drogarias & 24 & $(2,7)$ & 920 & $(1,4)$ & & & 514 & $(6,7)$ & \\
\hline Total & 893 & - & 67.361 & - & & & .759 & - & \\
\hline
\end{tabular}

Estab $=$ Estabelecimento

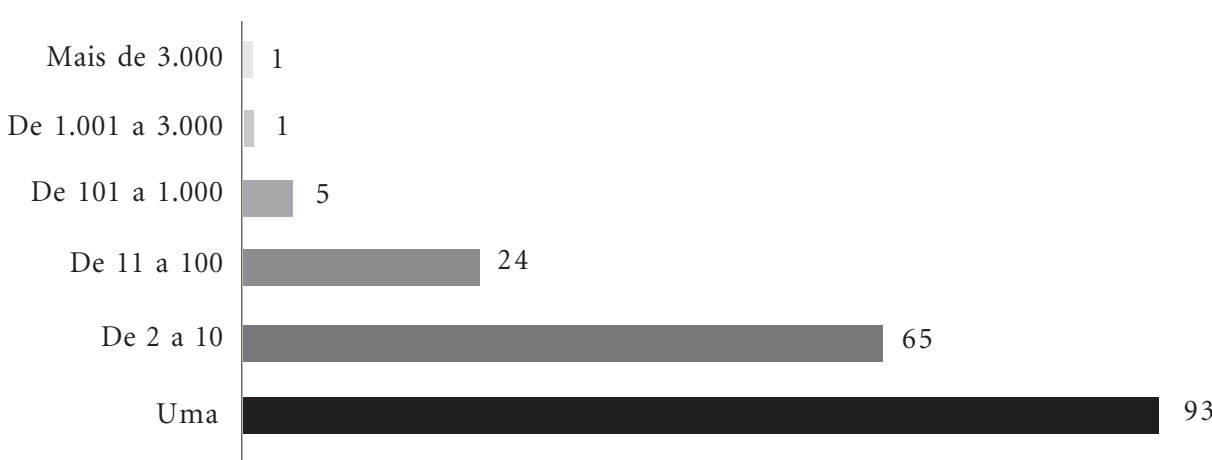

Gráfico 1. Frequência absoluta de profissionais que prescreveram em cada uma das categorias, estabelecidas de acordo com o número de prescrições dispensadas por CRM

bela 4), de acordo com CRM, não se observou a mesma relação entre prescritor e estabelecimento. O estabelecimento 11 dispensou, em números absolutos, a maior quantidade -14 do mesmo profissional ("J"), durante o ano pesquisado. O médico "J" teve 53 , das 78 prescrições emitidas, dispensadas em drogarias (correspondendo a $67,9 \%$ do total deste profissional). O médico "S" apresentou 84,6\% de suas prescrições dispensadas naquele tipo de estabelecimento. Porém, este médico emitiu somente 26 receitas de anorexígenos, das quais 22 foram dispensadas em drogarias. Para o profissional "S", o maior número de dispensações (em número de 6) ocorreu na drogaria 15.

Considerando-se os profissionais com maior número de prescrições (100 ou mais), observouse que sete deles foram responsáveis por 6.492 dispensações no ano de 2009, correspondendo a $83,7 \%$ do total. Todos os sete profissionais tiveram mais de $91 \%$ de suas prescrições dispensadas em farmácias, sendo que o médico com maior número de prescrições teve $100 \%$ das 3.535 prescrições dispensadas em farmácias (99,5\% em único estabelecimento).

O consumo anual de cada fármaco está descrito na Tabela 2, no campo “Total”, referente à soma das dispensações durante o ano, em miligramas. Como população, utilizou-se o dado do censo do Instituto Brasileiro de Geografia e Estatística (IBGE), em 201011 - 517.872 habitantes. Como Juiz de Fora é cidade polo de uma microrregião, também foi calculada a DDD/mil hab/dia da microrregião, utilizando o valor de 730.264 habitantes. 
Tabela 3. Prescrições dispensadas em farmácias, por CRM (20 profissionais com maior número de prescrições).

\begin{tabular}{|c|c|c|c|c|c|c|c|c|c|c|c|}
\hline \multirow{3}{*}{ CRM } & \multirow{3}{*}{$\begin{array}{c}\text { Total de } \\
\text { prescrições }\end{array}$} & \multicolumn{10}{|c|}{ Estabelecimentos } \\
\hline & & \multicolumn{2}{|c|}{1} & \multicolumn{2}{|c|}{2} & \multicolumn{2}{|c|}{3} & \multicolumn{2}{|r|}{4} & \multicolumn{2}{|c|}{5} \\
\hline & & $\mathbf{N}$ & $(\%)$ & $\mathbf{N}$ & $(\%)$ & $\mathrm{N}$ & $(\%)$ & $\mathbf{N}$ & $(\%)$ & $\mathrm{N}$ & $(\%)$ \\
\hline A & 3535 & 3518 & $(99,5)$ & 15 & $(0,4)$ & 0 & $(0,0)$ & 0 & $(0,0)$ & 0 & $(0,0)$ \\
\hline $\mathrm{B}$ & 1336 & 50 & $(3,8)$ & 791 & $(59,7)$ & 234 & $(17,6)$ & 143 & $(10,8)$ & 57 & $(4,3)$ \\
\hline $\mathrm{C}$ & 763 & 109 & $(15,1)$ & 311 & $(43,0)$ & 134 & $(18,5)$ & 136 & $(18,8)$ & 10 & $(1,4)$ \\
\hline $\mathrm{D}$ & 261 & 5 & $(2,0)$ & 150 & $(60,0)$ & 6 & $(2,4)$ & 70 & $(28,0)$ & 16 & $(6,4)$ \\
\hline $\mathrm{E}$ & 246 & 3 & $(1,2)$ & 10 & $(4,1)$ & 5 & $(2,0)$ & 1 & $(0,4)$ & 223 & $(91,4)$ \\
\hline $\mathrm{F}$ & 217 & 4 & $(2,0)$ & 160 & $(80,0)$ & 16 & $(8,0)$ & 12 & $(6,0)$ & 3 & $(1,5)$ \\
\hline G & 134 & 7 & $(5,7)$ & 77 & $(62,6)$ & 14 & $(11,4)$ & 9 & $(7,3)$ & 0 & $(0,0)$ \\
\hline $\mathrm{H}$ & 88 & 5 & $(10,6)$ & 29 & $(61,7)$ & 7 & $(14,9)$ & 6 & $(12,8)$ & 0 & $(0,0)$ \\
\hline I & 85 & 0 & $(0,0)$ & 0 & $(0,0)$ & 0 & $(0,0)$ & 0 & $(0,0)$ & 0 & $(0,0)$ \\
\hline $\mathrm{J}$ & 78 & 0 & $(0,0)$ & 6 & $(24,0)$ & 9 & $(36,0)$ & 3 & $(12,0)$ & 1 & $(4,0)$ \\
\hline $\mathrm{K}$ & 71 & 0 & $(0,0)$ & 36 & $(51,4)$ & 11 & $(15,7)$ & 0 & $(0,0)$ & 0 & $(0,0)$ \\
\hline $\mathrm{L}$ & 65 & 0 & $(0,0)$ & 13 & $(48,1)$ & 1 & $(3,7)$ & 3 & $(11,1)$ & 1 & $(3,7)$ \\
\hline $\mathrm{M}$ & 46 & 1 & $(2,2)$ & 0 & $(0,0)$ & 45 & $(97,8)$ & 0 & $(0,0)$ & 0 & $(0,0)$ \\
\hline $\mathrm{N}$ & 41 & 0 & $(0,0)$ & 11 & $(83,3)$ & 0 & $(0,0)$ & 1 & $(8,3)$ & 0 & $(0,0)$ \\
\hline $\mathrm{O}$ & 40 & 0 & $(0,0)$ & 12 & $(36,1)$ & 11 & $(30,6)$ & 5 & $(13,9)$ & 0 & $(0,0)$ \\
\hline $\mathrm{P}$ & 38 & 0 & $(0,0)$ & 7 & $(33,3)$ & 1 & $(6,7)$ & 0 & $(0,0)$ & 0 & $(0,0)$ \\
\hline Q & 35 & 1 & $(3,8)$ & 10 & $(38,5)$ & 6 & $(23,1)$ & 7 & $(26,9)$ & 0 & $(0,0)$ \\
\hline $\mathrm{R}$ & 34 & 1 & $(2,6)$ & 32 & $(94,7)$ & 0 & $(0,0)$ & 0 & $(0,0)$ & 0 & $(0,0)$ \\
\hline$S$ & 26 & 0 & $(0,0)$ & 0 & $(0,0)$ & 0 & $(0,0)$ & 0 & $(0,0)$ & 1 & $(25,0)$ \\
\hline $\mathrm{T}$ & 25 & 1 & $(4,8)$ & 16 & $(81,0)$ & 1 & $(4,8)$ & 1 & $(4,8)$ & 0 & $(0,0)$ \\
\hline Outros ${ }^{*}$ & 595 & 26 & $(6,6)$ & 189 & $(47,8)$ & 32 & $(8,1)$ & 40 & $(10,1)$ & 14 & $(3,5)$ \\
\hline Total & 7759 & 3731 & - & 1875 & - & 533 & - & 437 & - & 326 & - \\
\hline \multirow{3}{*}{ CRM } & Total de & \multicolumn{10}{|c|}{ Estabelecimentos } \\
\hline & prescrições & \multicolumn{2}{|c|}{6} & \multicolumn{2}{|c|}{7} & \multicolumn{2}{|c|}{8} & \multirow{2}{*}{\multicolumn{2}{|c|}{ Soma }} & \multirow{2}{*}{\multicolumn{2}{|c|}{$\begin{array}{c}\text { (\% do total de } \\
\text { prescrições) }\end{array}$}} \\
\hline & & $\mathrm{N}$ & $(\%)$ & $\mathbf{N}$ & $(\%)$ & $\mathbf{N}$ & $(\%)$ & & & & \\
\hline A & 3535 & 2 & $(0,1)$ & 0 & $(0,0)$ & 0 & $(0,0)$ & & \multicolumn{2}{|c|}{$(100,0)$} \\
\hline B & 1336 & 50 & $(3,8)$ & 0 & $(0,0)$ & 1 & $(0,1)$ & \multicolumn{2}{|r|}{1326} & $(99$ & \\
\hline $\mathrm{C}$ & 763 & 20 & $(2,8)$ & 1 & $(0,1)$ & 2 & $(0,3)$ & & $\begin{array}{r}1326 \\
723\end{array}$ & $(94$ & \\
\hline $\mathrm{D}$ & 261 & 2 & $(0,8)$ & 0 & $(0,0)$ & 1 & $(0,4)$ & & 250 & $(95$ & \\
\hline $\mathrm{E}$ & 246 & 2 & $(0,8)$ & 0 & $(0,0)$ & 0 & $(0,0)$ & & $\begin{array}{l}250 \\
244\end{array}$ & $(99$ & \\
\hline $\mathrm{F}$ & 217 & 4 & $(2,0)$ & 1 & $(0,5)$ & 0 & $(0,0)$ & & $\begin{array}{l}244 \\
200\end{array}$ & $(92$ & \\
\hline G & 134 & 16 & $(13,0)$ & 0 & $(0,0)$ & 0 & $(0,0)$ & & $\begin{array}{l}200 \\
123\end{array}$ & $(91$ & \\
\hline $\mathrm{H}$ & 88 & 0 & $(0,0)$ & 0 & $(0,0)$ & 0 & $(0,0)$ & & $\begin{array}{r}123 \\
47\end{array}$ & $(53$ & \\
\hline I & 85 & 0 & $(0,0)$ & 85 & $(100,0)$ & 0 & $(0,0)$ & & 85 & $(100$ & \\
\hline $\mathrm{J}$ & 78 & 0 & $(0,0)$ & 0 & $(0,0)$ & 6 & $(24,0)$ & & $\begin{array}{l}03 \\
25\end{array}$ & $(32$ & \\
\hline K & 71 & 23 & $(32,9)$ & 0 & $(0,0)$ & 0 & $(0,0)$ & & 70 & $(98$ & \\
\hline $\mathrm{L}$ & 65 & 7 & $(25,9)$ & 0 & $(0,0)$ & 2 & $(7,4)$ & & 27 & $(41$, & \\
\hline $\mathrm{M}$ & 46 & 0 & $(0,0)$ & 0 & $(0,0)$ & 0 & $(0,0)$ & & 46 & $(100$ & \\
\hline $\mathrm{N}$ & 41 & 0 & $(0,0)$ & 1 & $(8,3)$ & 0 & $(0,0)$ & & $\begin{array}{l}40 \\
13\end{array}$ & $(31$ & \\
\hline $\mathrm{O}$ & 40 & 7 & $(19,4)$ & 0 & $(0,0)$ & 0 & $(0,0)$ & & 35 & $(87$, & \\
\hline $\mathrm{P}$ & 38 & 0 & $(0,0)$ & 0 & $(0,0)$ & 9 & $(60,0)$ & & 17 & $(44$ & \\
\hline Q & 35 & 0 & $(0,0)$ & 1 & $(3,8)$ & 1 & $(3,8)$ & & 26 & $(74$ & \\
\hline $\mathrm{R}$ & 34 & 0 & $(0,0)$ & 1 & $(2,6)$ & 0 & $(0,0)$ & & 34 & $(100$ & \\
\hline$S$ & 26 & 0 & $(0,0)$ & 0 & $(0,0)$ & 3 & $(75,0)$ & & 4 & $(15$ & \\
\hline $\mathrm{T}$ & 25 & 0 & $(0,0)$ & 0 & $(0,0)$ & 1 & $(4,8)$ & & 20 & $(80$ & \\
\hline Outros* & 595 & 53 & $(13,4)$ & 2 & $(0,5)$ & 39 & $(9,9)$ & & 395 & $(66$ & \\
\hline Total & 7759 & 186 & - & 92 & - & 65 & - & & 7245 & - & \\
\hline
\end{tabular}

*169 profissionais, não incluídos entre aqueles com maior número de prescrições. 
Tabela 4. Prescrições aviadas em drogarias, de acordo com CRM, abrangendo os 20 profissionais com maior número de dispensações.

\begin{tabular}{|c|c|c|c|c|c|c|c|c|c|c|c|}
\hline \multirow{3}{*}{ CRM } & \multirow{3}{*}{$\begin{array}{c}\text { Total de } \\
\text { prescrições }\end{array}$} & \multicolumn{10}{|c|}{ Estabelecimentos } \\
\hline & & \multicolumn{2}{|c|}{9} & \multicolumn{2}{|c|}{10} & \multicolumn{2}{|c|}{11} & \multicolumn{2}{|c|}{12} & \multicolumn{2}{|c|}{13} \\
\hline & & $\mathrm{N}$ & $(\%)$ & $\mathbf{N}$ & $(\%)$ & $\mathbf{N}$ & $(\%)$ & $\mathrm{N}$ & $(\%)$ & $\mathbf{N}$ & $(\%)$ \\
\hline A & 3535 & 0 & $(0,0)$ & 0 & $(0,0)$ & 0 & $(0,0)$ & 0 & $(0,0)$ & 0 & $(0,0)$ \\
\hline B & 1336 & 0 & $(0,0)$ & 2 & $(0,1)$ & 2 & $(0,1)$ & 3 & $(0,2)$ & 0 & $(0,0)$ \\
\hline $\mathrm{C}$ & 763 & 6 & $(0,8)$ & 6 & $(0,8)$ & 3 & $(0,4)$ & 4 & $(0,5)$ & 4 & $(0,5)$ \\
\hline $\mathrm{D}$ & 261 & 3 & $(1,1)$ & 6 & $(2,3)$ & 0 & $(0,0)$ & 1 & $(0,4)$ & 0 & $(0,0)$ \\
\hline $\mathrm{E}$ & 246 & 2 & $(0,8)$ & 0 & $(0,0)$ & 0 & $(0,0)$ & 0 & $(0,0)$ & 0 & $(0,0)$ \\
\hline F & 217 & 4 & $(1,8)$ & 0 & $(0,0)$ & 1 & $(0,5)$ & 2 & $(0,9)$ & 4 & $(1,8)$ \\
\hline G & 134 & 0 & $(0,0)$ & 1 & $(0,7)$ & 2 & $(1,5)$ & 0 & $(0,0)$ & 2 & $(1,5)$ \\
\hline $\mathrm{H}$ & 88 & 7 & $(8,0)$ & 3 & $(3,4)$ & 4 & $(4,5)$ & 3 & $(3,4)$ & 11 & $(12,5)$ \\
\hline I & 85 & 0 & $(0,0)$ & 0 & $(0,0)$ & 0 & $(0,0)$ & 0 & $(0,0)$ & 0 & $(0,0)$ \\
\hline $\mathrm{J}$ & 78 & 5 & $(6,4)$ & 11 & $(14,1)$ & 14 & $(17,9)$ & 4 & $(5,1)$ & 6 & $(7,7)$ \\
\hline K & 71 & 0 & $(0,0)$ & 0 & $(0,0)$ & 0 & $(0,0)$ & 0 & $(0,0)$ & 0 & $(0,0)$ \\
\hline $\mathrm{L}$ & 65 & 2 & $(3,1)$ & 3 & $(4,6)$ & 10 & $(15,4)$ & 4 & $(6,2)$ & 5 & $(7,7)$ \\
\hline $\mathrm{M}$ & 46 & 0 & $(0,0)$ & 0 & $(0,0)$ & 0 & $(0,0)$ & 0 & $(0,0)$ & 0 & $(0,0)$ \\
\hline $\mathrm{N}$ & 41 & 5 & $(12,2)$ & 4 & $(9,8)$ & 2 & $(4,9)$ & 4 & $(9,8)$ & 5 & $(12,2)$ \\
\hline $\mathrm{O}$ & 40 & 0 & $(0,0)$ & 2 & $(5,0)$ & 1 & $(2,5)$ & 0 & $(0,0)$ & 0 & $(0,0)$ \\
\hline $\mathrm{P}$ & 38 & 5 & $(13,2)$ & 6 & $(15,8)$ & 2 & $(5,3)$ & 3 & $(7,9)$ & 1 & $(2,6)$ \\
\hline Q & 35 & 0 & $(0,0)$ & 0 & $(0,0)$ & 2 & $(5,7)$ & 1 & $(2,9)$ & 3 & $(8,6)$ \\
\hline $\mathrm{R}$ & 34 & 0 & $(0,0)$ & 0 & $(0,0)$ & 0 & $(0,0)$ & 0 & $(0,0)$ & 0 & $(0,0)$ \\
\hline S & 26 & 5 & $(19,2)$ & 2 & $(7,7)$ & 0 & $(0,0)$ & 4 & $(15,4)$ & 3 & $(11,5)$ \\
\hline $\mathrm{T}$ & 25 & 0 & $(0,0)$ & 2 & $(8,0)$ & 0 & $(0,0)$ & 0 & $(0,0)$ & 1 & $(4,0)$ \\
\hline Outros* & 595 & 46 & $(7,7)$ & 34 & $(5,7)$ & 27 & $(4,5)$ & 36 & $(6,1)$ & 12 & $(2,0)$ \\
\hline Total & 7759 & 90 & & 82 & & 70 & & 69 & & 57 & \\
\hline
\end{tabular}

\begin{tabular}{|c|c|c|c|c|c|c|c|c|c|}
\hline \multirow{3}{*}{ CRM } & \multirow{3}{*}{$\begin{array}{c}\text { Total de } \\
\text { prescrições }\end{array}$} & \multicolumn{8}{|c|}{ Estabelecimentos } \\
\hline & & \multicolumn{2}{|c|}{14} & \multicolumn{2}{|c|}{15} & \multicolumn{2}{|c|}{16} & \multirow{2}{*}{ Soma } & \multirow{2}{*}{$\begin{array}{c}\text { (\% do total de } \\
\text { prescrições) }\end{array}$} \\
\hline & & $\mathbf{N}$ & $(\%)$ & $\mathbf{N}$ & $(\%)$ & $\mathbf{N}$ & $(\%)$ & & \\
\hline A & 3535 & 0 & $(0,0)$ & 0 & $(0,0)$ & 0 & $(0,0)$ & 0 & $(0,0)$ \\
\hline B & 1336 & 1 & $(0,1)$ & 1 & $(0,1)$ & 1 & $(0,1)$ & 10 & $(0,7)$ \\
\hline $\mathrm{C}$ & 763 & 9 & $(1,2)$ & 5 & $(0,7)$ & 3 & $(0,4)$ & 40 & $(5,2)$ \\
\hline $\mathrm{D}$ & 261 & 0 & $(0,0)$ & 0 & $(0,0)$ & 1 & $(0,4)$ & 11 & $(4,2)$ \\
\hline $\mathrm{E}$ & 246 & 0 & $(0,0)$ & 0 & $(0,0)$ & 0 & $(0,0)$ & 2 & $(0,8)$ \\
\hline $\mathrm{F}$ & 217 & 0 & $(0,0)$ & 5 & $(2,3)$ & 1 & $(0,5)$ & 17 & $(7,8)$ \\
\hline G & 134 & 1 & $(0,7)$ & 1 & $(0,7)$ & 4 & $(3,0)$ & 11 & $(8,2)$ \\
\hline $\mathrm{H}$ & 88 & 3 & $(3,4)$ & 5 & $(5,7)$ & 5 & $(5,7)$ & 41 & $(46,6)$ \\
\hline I & 85 & 0 & $(0,0)$ & 0 & $(0,0)$ & 0 & $(0,0)$ & 0 & $(0,0)$ \\
\hline $\mathrm{J}$ & 78 & 7 & $(9,0)$ & 2 & $(2,6)$ & 4 & $(5,1)$ & 53 & $(67,9)$ \\
\hline K & 71 & 1 & $(1,4)$ & 0 & $(0,0)$ & 0 & $(0,0)$ & 1 & $(1,4)$ \\
\hline $\mathrm{L}$ & 65 & 3 & $(4,6)$ & 7 & $(10,8)$ & 4 & $(6,2)$ & 38 & $(58,5)$ \\
\hline $\mathrm{M}$ & 46 & 0 & $(0,0)$ & 0 & $(0,0)$ & 0 & $(0,0)$ & 0 & $(0,0)$ \\
\hline $\mathrm{N}$ & 41 & 0 & $(0,0)$ & 6 & $(14,6)$ & 2 & $(4,9)$ & 28 & $(68,3)$ \\
\hline $\mathrm{O}$ & 40 & 1 & $(2,5)$ & 0 & $(0,0)$ & 1 & $(2,5)$ & 5 & $(12,5)$ \\
\hline $\mathrm{P}$ & 38 & 0 & $(0,0)$ & 1 & $(2,6)$ & 1 & $(2,6)$ & 19 & $(50,0)$ \\
\hline $\mathrm{Q}$ & 35 & 1 & $(2,9)$ & 3 & $(8,6)$ & 1 & $(2,9)$ & 11 & $(31,4)$ \\
\hline $\mathrm{R}$ & 34 & 0 & $(0,0)$ & 0 & $(0,0)$ & 0 & $(0,0)$ & 0 & $(0,0)$ \\
\hline S & 26 & 2 & $(7,7)$ & 6 & $(23,1)$ & 0 & $(0,0)$ & 22 & $(84,6)$ \\
\hline $\mathrm{T}$ & 25 & 0 & $(0,0)$ & 2 & $(8,0)$ & 0 & $(0,0)$ & 5 & $(20,0)$ \\
\hline Outros & 595 & 24 & $(4,0)$ & 6 & $(1,0)$ & 15 & $(2,5)$ & 200 & $(33,7)$ \\
\hline Total & 7759 & 53 & & 50 & & 43 & & 514 & \\
\hline
\end{tabular}

* 169 profissionais, não incluídos entre aqueles com maior número de prescrições. 
Conforme orienta ATC, os valores para DDD são de $75 \mathrm{mg}$ para anfepramona e $1 \mathrm{mg}$ para mazindol. Para femproporex, não há DDD estabelecida, nem o fármaco está cadastrado no sistema ATC $^{16}$. Para fins de comparação, mantevese a DDD utilizada pela Anvisa no cálculo do femproporex $(25 \mathrm{mg})$.

Para cada fármaco, os cálculos foram efetuados para o município, sendo identificados como "JF"; e para a microrregião, sendo identificados como "Micro".

Sendo assim, para femproporex, obteve-se a seguinte DDD/mil hab/dia:

$\underset{\text { habitantes/dia (JF) }}{\mathrm{DDD} / \mathrm{mil}}=\frac{3.218 .645 \times 1.000}{25 \times 517.872 \times 365}=0,68$

$\underset{\text { habitantes } / \text { dia }}{\mathrm{DDD} / \mathrm{mil}}=\frac{3.218 .645 \times 1.000}{25 \times 730.264 \times 365}=0,68$ (Micro)

O valor, de 2009, no relatório da Anvisa, foi de 0,62 mg/1.000 hab/dia.

Para anfepramona, obteve-se a seguinte DDD/ mil hab/dia:

$\underset{\text { habitantes/dia (JF) }}{\mathrm{DDD} / \mathrm{mil}}=\frac{13.746 .025 \times 1.000}{75 \times 517.872 \times 365}=0,97$

$\underset{\mathrm{DDD} / \mathrm{mil}}{\text { habitantes } / \mathrm{dia}} \quad=\frac{13.746 .025 \times 1.000}{75 \times 730.264 \times 365}=0,69$

(Micro)

O valor de 2009, no relatório da Anvisa, foi de $0,61 \mathrm{mg} / 1.000 \mathrm{hab} / \mathrm{dia}$.

Para mazindol, obteve-se a seguinte DDD/mil hab/dia:

$\underset{\text { habitantes } / \text { dia }(J F)}{\mathrm{DDD} / \mathrm{mil}}=\frac{67.361 \times 1.000}{1 \times 517.872 \times 365}=0,36$

$\underset{\begin{array}{c}\mathrm{DDD} / \mathrm{mil} \\ \text { habitantes} / \mathrm{dia} \\ (\text { Micro })\end{array}}{\mathrm{M}}=\frac{67.361 \times 1.000}{1 \times 730.264 \times 365}=0,25$

O valor de 2009, no relatório da Anvisa, foi de $0,035 \mathrm{mg} / 1.000 \mathrm{hab} /$ dia.

\section{Limitações do estudo}

Juiz de Fora é a cidade polo da microrregião da Zona da Mata. Portanto, pacientes de outros municípios se tratam na cidade, pela ausência de especialistas nas localidades vizinhas, fato que pode ter contribuído para aumentar a quantidade de anorexígenos dispensada no município.

Não foram analisadas as prescrições e, sim, os dados constantes do SNGPC e da RMNB2. Portanto, irregularidades no preenchimento das notificações não puderam ser avaliadas. Como essas irregularidades podem existir, não é possível aferir a regularidade das informações repassadas aos sistemas de informações pesquisa$\operatorname{dos}^{17,18}$. Além disso, não é possível verificar se as prescrições se originaram em consultório particular ou em unidades de atendimento do Sistema Único de Saúde (SUS).

O médico pode não ter cadastrado sua especialidade no CRM. Como o registro é necessário apenas para fins de divulgação, só há exigência legal para tal se houver publicidade. Existe a possibilidade de um profissional ser especialista e não estar cadastrado no CRM.

Não foram analisados fatores comerciais que poderiam interferir no padrão de consumo e dispensação: preço, localização dos estabelecimentos (proximidade dos consultórios) e propaganda (publicidade em mídia).

\section{Discussão}

Erros no preenchimento de prescrições são informados em diversos estudos ${ }^{18-21}$. Porém, erros nas informações da Relação Mensal entregue à VISA Municipal não haviam sido relatados. A existência de campos preenchidos com o CRM de médicos falecidos, transferidos ou com inscrições canceladas é motivo de investigação. Cabe averiguar se houve falsificação de talonário, erro de digitação ou fraude no preenchimento.

No presente trabalho, anfepramona foi o fármaco mais prescrito, atingindo 55,2\% das prescrições, versus $33,4 \%$ de prescrições de femproporex. Resultado similar foi obtido por estudos realizados nos estados do Pará, Rio Grande do Sul e Paraná, respectivamente ${ }^{21-23}$. Borges et al..$^{21}$, em análise de 49 estabelecimentos privados de uma rede de farmácias e drogarias em Belém, evidenciaram maior consumo de anorexígenos de menor preço de venda em drogarias. Já em estudos de Carneiro et al. ${ }^{17}$ e Cole et al. ${ }^{18}$, houve predomínio de prescrições de femproporex (seguido por anfepramona).

Um único estabelecimento foi responsável por $47,6 \%$ de todas as dispensações no período analisado. Somando-se ao segundo estabelecimento 
de maior movimento, esse valor chega a $71,5 \%$ do total. Levando em consideração que a distância entre os estabelecimentos é pequena, pois, das oito farmácias selecionadas, sete se localizavam no centro da cidade (em raio de aproximadamente 700 metros), o predomínio desses estabelecimentos deve ser avaliado: existe um diferencial que justifique o maior volume de vendas?

Médicos sem especialidade registrada no CRM constituíram o grupo de maiores prescritores, sendo responsáveis por $57 \%$ de todas as dispensações no período, seguidos pelos especialistas em Endocrinologia (35\%) e Cardiologia $(3,2 \%)$. Apareceram especialidades pouco usuais em se tratando de manejo de obesidade, como Oftalmologia e Radiologia. Dados semelhantes foram encontrados no Relatório da Anvisa $(2010)^{10}$ e no estudo de Carneiro et al. ${ }^{17}$, em que figuraram especialidades como Medicina do Tráfego e até áreas como Medicina Veterinária. Nesse aspecto, o relatório "Resultados do SNGPC 2009 " 24 evidencia que, entre os dez profissionais com maior número de prescrições de anfepramona no Brasil, estão um Ginecologista e um Gastroenterologista. O profissional com maior número de prescrições de femproporex do país é médico dermatologista, e, entre os dez profissionais com maior número de prescrições de mazindol, há um médico pediatra. Sabe-se que todo médico pode atuar prescrevendo qualquer tipo de medicamento, mas o tratamento farmacológico da obesidade envolve uso de medicamentos com grandes potenciais para tolerância e dependência, além de importantes contraindicações e efeitos colaterais. Se um determinado profissional prescreve esse tipo de substância em grande quantidade, pode-se levantar a suspeita de mau uso ou desvio de prescrições, fatos que precisam ser averiguados e devidamente punidos. Na presente pesquisa, os médicos que prescreveram grandes quantidades de anorexígenos psicotrópicos durante 2009 foram poucos, sugerindo que essa prática não é generalizada. A prescrição de anorexígenos por especialistas de diversas áreas pode ser compreensível sob o ponto de vista da ação integral em saúde, principalmente no contexto da atenção primária em saúde, em que médicos de qualquer especialidade e mesmo recém-formados ainda sem especialização trabalham no atendimento a populações adstritas.

Os prescritores desempenham papel fundamental no uso racional desses medicamentos. É ainda indispensável o aprimoramento das ações de vigilância sanitária, com o propósito de aperfeiçoar o controle e a fiscalização dessas substân- cias, mediando seu comércio e contribuindo para a construção de um uso e um acesso mais racionais. Intervenções no sentido não apenas de controlar, mas de informar médicos, farmacêuticos, enfermeiros e pacientes, parecem ser as formas de atuação mais promissoras ${ }^{23}$.

No presente trabalho, os resultados sugerem prática médica inadequada, pois o profissional com maior número de prescrições (entre 189 médicos) foi responsável por $45,1 \%$ das prescrições de anorexígenos e teve $99,5 \%$ de suas prescrições aviadas em um único estabelecimento. Cabe à Vigilância Sanitária verificar se as dispensações ocorreram de maneira lícita e, ao Conselho Regional de Medicina, se as prescrições obedeceram aos preceitos éticos da profissão.

Uma estratégia importante para fortalecer o monitoramento do consumo dessas substâncias pelos órgãos de vigilância sanitária inclui o uso de indicadores de acompanhamento da situação e a análise sistemática das inspeções, a ser viabilizada por meio de sistema informatizado, que dê apoio às auditorias fiscais e ao gerenciamento das informações coletadas. Após esse período de implantação do SNGPC, tal sistema pode se tornar ferramenta importante para o registro do consumo de psicotrópicos, possibilitando o acesso e o cruzamento das informações de maneira mais rápida e eficaz.

Espera-se aumento na eficácia das campanhas para o uso racional de medicamentos, com atividades dirigidas aos principais problemas identificados nas prescrições de psicotrópicos anorexígenos: erros de preenchimento nas prescrições e/ou relações de consumo enviadas à VISA, grande número de prescrições por um único profissional, associação entre os profissionais e os estabelecimentos farmacêuticos e doses acima do recomendado pelas diretrizes médicas e resoluções das instâncias regulatórias.

Em 23 de fevereiro de 2011, a Anvisa realizou audiência pública, que discutiu a proposta de retirada do mercado brasileiro dos inibidores de apetite, a exemplo da decisão tomada pela União Europeia e pelos Estados Unidos. A iniciativa visa garantir à população que os medicamentos com venda permitida no Brasil sejam seguros. O cancelamento do registro e, consequentemente, a proibição de comercialização desses produtos, apesar de combatido por determinados setores médicos e farmacêuticos, são uma realidade em outros países. Anfepramona, femproporex e mazindol não são comercializados na Europa desde 2000. Femproporex nunca recebeu autorização de FDA para ser vendido nos Estados Unidos. A Sibutra- 
mina, apesar de não estar incluída no estudo, teve o registro cancelado na Europa, em janeiro de 2010. Foi retirada de comercialização, voluntariamente, pelo fabricante em outubro de 2010, em Canadá, Austrália e Estados Unidos, e em novembro do mesmo ano, no Brasil ${ }^{25}$.

O sucesso das estratégias apontadas requer uma ação conjunta da vigilância sanitária, dos conselhos profissionais das categorias médica e farmacêutica e dos estabelecimentos de ensino superior, fortalecendo o combate ao uso excessivo e antiético das substâncias psicotrópicas anorexígenas, buscando a agilização dos mecanismos de monitoramento do consumo, bem como incrementando a promoção da racionalidade de seu uso. Verifica-se a necessidade de melhorar a qualidade dos serviços médicos e farmacêuticos prestados à comunidade e de ampliar a conscientização dos usuários sobre os riscos inerentes ao uso abusivo e desnecessário dessas substâncias. Ressalta-se, ainda, a importância que a distribuição e o consumo deste tipo de fármaco assu- mem, tendo de um lado o mercado farmacêutico e de outro a saúde pública, onerada pelos problemas acarretados pelo uso irracional desses medicamentos.

O SNGPC se mostra um produto confiável e capaz de cumprir os objetivos, desde que implantado e fiscalizado com rigor. Encontrou-se um número pequeno, porém considerável, de prescrições com erros. Alguns podem ter sido de digitação, mas, quando eles se repetem no mesmo estabelecimento, levanta-se a suspeita de má prática. Informar os gestores da saúde sobre a gravidade da situação no município permitirá à Vigilância Sanitária local embasar a tomada de decisões e a programação de ações educativas locais.

$O$ resultado do presente trabalho aponta o uso de psicotrópicos anorexígenos como um problema relevante de saúde coletiva e indica a necessidade de reavaliação das políticas até então adotadas em relação a esses produtos em nosso país, bem como dos critérios de monitoramento de sua dispensação e consumo.

\section{Colaboradores}

ELM Martins e MPH Amaral contribuíram em todas as etapas do trabalho: concepção, coleta e análise dos dados, redação e revisão do artigo. MBC Ferreira e AE Mendonça participaram da concepção, análise dos dados, redação e revisão do artigo. MCS Pereira, DC Pereira, PM Ribeiro e LP Brumano participaram da coleta e análise de dados. 


\section{Referências}

1. Faria AM, Mancini MC, Melo ME, Cercato C, Halpern A. Progressos recentes e novas perspectivas em farmacoterapia da obesidade. Arq Bras Endocrinol Metabol 2010; 54(6):516-529.

2. Lozano MJL, Gonzales AS. Actualización en Obesidad. Cad Aten Primaria 2010; 17:101-107.

3. World Health Organization (WHO). Consultation on Obesity. Preventing and Managing the Global Epidemic. Geneva: WHO; 1998.

4. Prentice AM, Jebb AS. Obesity in Britain: gluttony or sloth. BMJ 1995; 311(7002):437439.

5. Associação Brasileira para o Estudo da Obesidade e da Síndrome Metabólica (ABESO). Diretrizes brasileiras de obesidade 2009/2010. 3a Edição. Itapevi, SP: AC Farmacêutica; 2009.

6. Brasil. Ministério da Saúde. Portaria nº. 344, de 12 de maio de 1998. Aprova o regulamento técnico sobre substâncias e medicamentos sujeitos a controle especial. Brasília, DF, Diário Oficial da União 1998; 12 maio.

7. Katzung BG. Farmacologia Básica e Clínica. 6a Edição. Rio de Janeiro: Guanabara Koogan; 1996.

8. Organização Mundial da Saúde (OMS). Preventing and managing the Global Epidemic of Obesity. Report of the World Health Organization Consultation of Obesity. Genebra: OMS; 2000.

9. Brasil. Ministério da Saúde. Agência Nacional de Vigilância Sanitária. Resolução RDC nº. 27, de 30 de março de 2007. Dispõe sobre o Sistema Nacional de Gerenciamento de Produtos Controlados SNGPC, estabelece a implantação do módulo para drogarias e farmácias e dá outras providências. Diário Oficial da União 2007; 30 mar.

10. Brasil. Ministério da Saúde. Agência Nacional de Vigilância Sanitária. Sistema Nacional de Gerenciamento de Produtos Controlados - SNGPC. [acessado 2011 jan 17]. 2010. Disponível em: http://www.anvisa. gov.br/hotsite/sngpc/apresenta.htm.

11. Brasil. Instituto Brasileiro de Geografia e Estatística (IBGE). Censo Populacional 2010. [acessado 2011 jan 10]. 2010. Disponível em: http://www.ibge.gov. br/ home/estatistica/populacao/censo2010/populacao_ por_municipio.shtm.

12. Brasil. Ministério da Saúde. Agência Nacional de Vigilância Sanitária. Resolução RDC nº . 13, de 26 de março de 2010. Dispõe sobre a atualização do Anexo I, Lista de Substâncias Entorpecentes, Psicotrópicas, Precursoras e Outras sob Controle Especial da Portaria SVS/MS n ${ }^{\circ} 344$, de 12 de maio de 1998 e dá outras providências. Diário Oficial da União 2010; 26 mar

13. Brasil. Ministério da Saúde. Agência Nacional de Vigilância Sanitária. Resolução RDC no ${ }^{\circ} .58$, de 5 de setembro de 2007. Dispões sobre o aperfeiçoamento do controle e fiscalização de substâncias psicotrópicas anorexígenas e dá outras providências. Diário Oficial da União 2007; 5 set.

14. Conselho Regional de Medicina de Minas Gerais. Cadastro. [site na Internet]. [acessado 2012 out 15]. Disponível em: www.crememg.org.br

15. Brasil. Ministério da Saúde. Portaria no 1.179 , de 17 de junho de 1996. Diário Oficial da União 1996; 18 jun
16. Organização Mundial da Saúde (OMS). World He alth Organization Collaborating Centre for Drug Statistics Methodology. Anatomical Therapeutic Chemical (ATC) Index with Defined Daily Doses (DDDs), 2010 [acessado 2011 jan 23]. 2010. Disponível em: http://www.whocc.no/atcddd.

17. Carneiro MFG, Guerra Júnior AA, Acúrio FA. Prescrição, dispensação e regulação do consumo de psicotrópicos anorexígenos em Belo Horizonte, Minas Gerais, Brasil. Cad Saude Publica 2008; 24(8):1763-1772.

18. Cole ER, Arpini AF, Andrade CR, Biancard, EF. Terapia farmacológica da obesidade: uma análise crítica e reflexiva das prescrições de catecolami nérgicos por uma farmácia de manipulação do município de Vila Velha, Espírito Santo. Rev Eletron Farm 2009; 6(4):10-61.

19. Noto AR, Carlini EA, Mastroianni PC, Alves VC, Galduróz JCF, Kuroiwa W, Csizmar J, Costa A, Faria MA, Hidalgo SR, Assis D, Nappo SA. Analysis of prescription and dispensation of psychotropic medications in two cities in the state of São Paulo, Brazil. Rev Bras Psiquiatr 2002; 24(2):68-73.

20. Camargo MDF, Lopes AM, Montebelo MI, Lopes LC. Avaliação da adesão às normas da portaria 344/ 98 SVS/MS utilizando notificações e receitas oriundas da cidade de Salto/SP. Rev Cienc Farm Basica Apl 2005; 26(2):131-137.

21. Borges GPM, Belo T, Vieira GMM, Vieira JRS. Análise dos receituários de medicamentos psicotrópicos anorexígenos em uma rede privada de farmácias de Belém - Pará. Rev Infarma 2008; 20(9):26-31.

22. Pizzol TSD, Branco MMN, Carvalho RMA, Pasqualotti A, Maciel EM, Migott AMB. Uso não-médico de medicamentos psicoativos entre escolares do ensino fundamental e médio no Sul do Brasil. Cad Saude Publica 2006; 22(1):109-115.

23. Silva M, Campesatto-Mella EA. Avaliação do uso de anorexígenos por acadêmicas de uma instituição de ensino superior em Maringá. Arq Cienc Saude Unipar 2008; 12(1):43-50.

24. Brasil. Ministério da Saúde. Agência Nacional de Vigilância Sanitária. Relatório SNGPC 2009. Brasília, DF: MS; 2010. [acessado 2011 jan 20]. Disponível em: http://www.anvisa.gov.br/hotsite/sngpc/ relatorio_2009.pdf.

25. Brasil. Ministério da Saúde (MS). Agência Nacional de Vigilância Sanitária. Eficácia e segurança dos medicamentos inibidores de apetite: nota técnica. Brasília, DF: MS; 2011.

Artigo apresentado em 01/08/2011

Aprovado em 04/08/2011

Versão final apresentada em 13/09/2012 